Pacific

Journal of

Mathematics

HILBERT SPACES OF TENSOR-VALUED HOLOMORPHIC FUNCTIONS ON THE UNIT BALL OF $\mathbb{C}^{n}$

Stephen Hwang, Yang LiU, and Genkai Zhang 


\title{
HILBERT SPACES OF TENSOR-VALUED HOLOMORPHIC FUNCTIONS ON THE UNIT BALL OF $\mathbb{C}^{n}$
}

\author{
Stephen Hwang, Yang Liu, and Genkai Zhang
}

\begin{abstract}
We study expansion of reproducing kernels for Hilbert spaces of holomorphic functions on the unit ball in $\mathbb{C}^{n}$ with values in the antisymmetric tensor of the tangent and the cotangent spaces. As an application we find the composition series for the analytic continuation of certain families of holomorphic discrete series.
\end{abstract}

\section{Introduction.}

The expansion of reproducing kernels of Bergman spaces of holomorphic functions on a domain $D$ in $\mathbb{C}^{n}$ is of considerable interests. If the domain admits an action of a compact group $K$, then naturally one would like to decompose the space of polynomials into irreducible subspaces of $K$ and expand the reproducing kernels in terms of the reproducing kernels of the finite dimensional subspaces. In [5] Hua found the expansion of the Bergman reproducing kernels on classical bounded symmetric domains. In that case the domain is a Hermitian symmetric space $D=G / K$, and the Bergman space forms also a unitary representation of the group $G$. Faraut and Koranyi [3] have recently found the expansion of weighted Bergman reproducing kernels on a general bounded symmetric domain and gave several implications. Since the appearance of that paper there have been more interests in the problem of finding the expansions.

In the previous papers [6] and [7] the authors studied the expansions of reproducing kernels for spaces of vector-valued holomorphic functions on classical matrix tube domains $U(n, n) /(U(n) \times U(n)), S p(n, \mathbb{R}) / U(n)$ and $O^{*}(2 n) / U(n)$ (for even integers $n$ ). The tangent spaces of the domains are $\mathbb{C}^{n} \otimes \mathbb{C}^{n}, \mathbb{C}^{n} \odot \mathbb{C}^{n}$ and $\mathbb{C}^{n} \wedge \mathbb{C}^{n}$ respectively. The holomorphic functions considered there take values in the space $\mathbb{C}^{n}$, which roughly speaking corresponds to the half of the tangent bundle, or the spin bundle. In this paper we take the simplest non-tube type domain, namely the unit ball $B^{n}$ in $\mathbb{C}^{n}$, and consider $G$-invariant Hilbert spaces of holomorphic functions with value in the antisymmetric $p$-tensor $\wedge^{p} V^{\prime}$ and $q$-tensor $\wedge^{q} V$ of the the cotangent space $V^{\prime}$ and respectively tangent space $V$; they are viewed as holomorphic sections of the respective bundles tensored with a line bundle. 
The spaces of cotangent vector-valued holomorphic functions appear naturally in the study of the harmonic analysis on those domains, in particular in Hodge theory. Also, if we consider the analytic continuation of a scalar discrete series on a non-tube domain, the unitary quotients in the composition series are equivalent to space of vector-valued holomorphic functions; see Remark 3.5. The other spaces that we consider in this paper are those of tangent vector-valued holomorphic functions. Some of those spaces can be realized as a relative discrete series of a weighted $L^{2}$-space on the unit ball $B^{n}$, cf. [13] and Remark 4.1. We find in this paper the expansion of the reproducing kernels of those spaces. As a direct consequence of our result we get the composition series for the analytic continuation of the holomorphic discrete series; for a general bounded symmetric domain, the composition series is still not known and quite a mystery. See also [4] for a detailed study of composition series of (scalar-valued) principal series representations of some classical groups.

We remark that the problem of finding the expansion of matrix-valued reproducing kernels has also been addressed in mathematical physics [10]. Furthermore, those spaces also provide some nontrivial and new examples of Hilbert modules over the function algebra $H^{\infty}\left(B^{n}\right)$, and might be of independent interests; see [1] and [2].

As it is pointed in [12] our question is also related to Capelli type and to the invariant differential operators on vector-valued functions on the symmetric domain; see further [11] Proposition 2.2. More precisely there corresponds to each $K$-invariant (matrix-valued) polynomial an invariant differential operators on vector-valued functions on the unit ball $B^{n}$. There are various open questions [11] concerning invariant differential operators on vector-valued functions on general bounded symmetric domains. We hope that our result will shed some light in understanding those problems.

\section{Preliminaries.}

Let $V=\mathbb{C}^{n}$ be the complex vector space equipped with the standard Hermitian inner product $(z, w)$. Let $B=B^{n}$ be the unit ball in $V$. The vectors in $V$ will be written as column vectors. Let $G=U(n, 1)$ be the Lie group of linear transformations of $\mathbb{C}^{n+1}$ that are isometric with respect to the sesquilinear form $\left|z_{1}\right|^{2}+\cdots+\left|z_{n}\right|^{2}-\left|z_{n+1}\right|^{2}$. We write any element of $G$ as a block matrix $\left(\begin{array}{ll}a & b \\ c & d\end{array}\right)$ under the decomposition of $\mathbb{C}^{n+1}=\mathbb{C}^{n}+\mathbb{C}$. It acts on the unit ball $B^{n}$ as biholomorphic mappings:

$$
g(z)=(a z+b)(c z+d)^{-1}, \quad g \in G, z \in B^{n} .
$$

$B^{n}$ is thus a symmetric space, $B^{n}=G / K$, where $K=U(n) \times U(1)$ is a maximal compact subgroup of $G$. 
Let $\mathfrak{g}_{0}$ and $\mathfrak{k}_{0}$ be the Lie algebra of $G$ and $K$ respectively. We denote by $\mathfrak{g}$ and $\mathfrak{k}$ their complexifications. Then $\mathfrak{g}=\mathfrak{g l}(n+1, \mathbb{C})$, the Lie algebra of the complex $(n+1) \times(n+1)$ complex matrices. Denote $E_{j, k}$ the matrix with 1 in the $(j, k)$-entry and 0 in the rest, and $E_{j}=E_{j, j}$. We choose a Cartan algebra $\mathfrak{h}$ to be the space of all diagonal matrices with basis $\left\{E_{j}, j=1, \ldots, n+1\right\}$, and let $\left\{\varepsilon_{j}, j=1, \ldots, n+1\right\}$ be the dual basis of $\mathfrak{h}^{\prime}, \varepsilon_{j}\left(E_{k}\right)=\delta_{j, k}$. The roots of $(\mathfrak{g}, \mathfrak{h})$ are then $\varepsilon_{j}-\varepsilon_{k}, j \neq k$, and $j, k=1,2, \ldots, n+1$. We choose an ordering of the roots by requiring $\varepsilon_{1}>\varepsilon_{2}>\cdots>\varepsilon_{n+1}$. Note that the compact roots are $\varepsilon_{j}-\varepsilon_{k}$ for $j, k=1,2 \ldots, n$.

The group $K$ acts on $V=\mathbb{C}^{n}$ via the defining action. We let $e_{1}, \ldots, e_{n}$ be the standard basis vectors of $V$. Let $V^{\prime}$ be the dual space of $V$ with the dual basis vectors $e_{1}^{\prime}, \ldots, e_{n}^{\prime}$, with the coadjoint action of $K . V^{\prime}$ will be realized as the space of row vectors. When viewed as holomorphic tangent vectors and forms we have $e_{j}=\frac{\partial}{\partial z_{j}}$ and $e_{j}^{\prime}=d z_{j}, j=1, \ldots, n$. The representations of $K$ on $V$ and $V^{\prime}$ are of highest weights $\varepsilon_{1}$ and $-\varepsilon_{n}$, with highest weight vectors $e_{1}$ and $e_{n}^{\prime}$, respectively.

Denote $\mathcal{P}=\mathcal{P}\left(B^{n}\right)$ the space of all holomorphic polynomials on $B^{n}$. There is a natural $K$-invariant Hermitian inner product on $\mathcal{P}$ whose completion is the so-called Fock space, namely

$$
(p, q)_{\mathcal{F}}=\left.p(\partial) q^{*}(z)\right|_{z=0},
$$

where the polynomial $q^{*}$ is obtained from $q$ by taking the complex conjugate of the coefficients of the monomials $z_{1}^{m_{1}} \ldots z_{n}^{m_{n}}$. As a representation space of $K$ it is decomposed into

$$
\mathcal{P}=\sum_{m=0}^{\infty} \mathcal{P}_{m}
$$

where $\mathcal{P}_{m}$ is the space of all homogeneous polynomials of degree $m$, and it has highest weight $-m \varepsilon_{n}$ and highest weight vector $v_{-m \varepsilon_{n}}=\frac{z_{n}^{m}}{\sqrt{m !}}$. The vector $v_{-m \varepsilon_{n}}$ then has norm 1 in the Fock space. With this inner product the whole space $\mathcal{P}$ has reproducing kernel $e^{(z, w)}$ and the spaces $\mathcal{P}_{m}$ have $\frac{(z, w)^{m}}{m !}$.

If $W$ is any finite-dimensional vector space with a $K$-invariant inner product we can equip as well the space $\mathcal{P}(W)=\mathcal{P} \otimes W$ of all $W$-valued polynomials on $B^{n}$ with the tensor of the Fock norm on $\mathcal{P}$ with the given norm in $W$.

We introduce now the Bergman operator on $V$

$$
B(z, w)=(1-(z, w))\left(1-z \otimes w^{*}\right),
$$

where $z \otimes w^{*}$ stands for the rank one operator given by $\left(z \otimes w^{*}\right)(v)=(v, w) z$. Viewed as a matrix it is

$$
B(z, w)=(1-(z, w))\left(1-z \bar{w}^{\prime}\right)
$$


where as before $w^{\prime}$ is the transpose of $w$. The Bergman metric at $z \in B^{n}$, when we identify the tangent space with $V$, is $\left(B(z, z)^{-1} u, v\right)$ for $u, v \in V$. We note that

$$
B(z, w)^{-1}=(1-(z, w))^{-2}\left(1-(z, w)+z \otimes w^{*}\right) .
$$

We denote $B^{t}(z, w)$ the dual of $B(z, w)$ acting on the dual space $V^{\prime}$ of $V$. When acting on vector in $v^{\prime} \in V^{\prime}$ it is

$$
B^{t}(z, w) v^{\prime}=(1-(z, w)) v^{\prime}\left(1-z \bar{w}^{\prime}\right) .
$$

\section{Hilbert spaces of holomorphic differential forms and the Dirichlet space.}

For $1 \leq p \leq n$ let $\wedge^{p} V^{\prime}$ be the anti-symmetric tensor of $V^{\prime}$, viewed as the constant holomorphic differential $p$-forms on $B^{n}$. The space $\wedge^{p} V^{\prime}$ is equipped with a natural Hermitian inner product induced from that of $V^{\prime}$, so that

$$
\left(d z_{1} \wedge \cdots \wedge d z_{p}, d z_{1} \wedge \cdots \wedge d z_{p}\right)=\frac{1}{p !} .
$$

Let $d m(z)$ be the Lebesgue measure on $B^{n}$ and

$$
d \mu_{\nu}(z)=\left(1-|z|^{2}\right)^{\nu-n-1} d m(z)
$$

be the weighted measure. For $\nu>n-1$ we consider the space $\mathcal{A}_{\nu, p}=$ $L_{a}^{2}\left(B^{n}, \wedge^{p} V^{\prime}, \mu_{\nu}\right)$ of $\wedge^{p} V^{\prime}$-valued holomorphic functions $f$ on $B^{n}$ so that

$$
\|f\|_{\nu}^{2}=\frac{1}{d_{\nu}} \int_{B^{n}}\left(\otimes^{p} B(z, z)^{t} f(z), f(z)\right) d \mu_{\nu}(z)<\infty
$$

where

$$
d_{\nu}=\pi^{n} \frac{\nu}{\nu+p} \frac{\Gamma(\nu+p-n)}{\Gamma(\nu+p)} .
$$

Here and after $\otimes^{p} B^{t}(z, z)$ on $\wedge^{p} V^{\prime}$ denotes the induced action of $B^{t}(z, z)$ on $V^{\prime}$. The inner product $\left(\otimes^{p} B(z, z)^{t} f(z), f(z)\right)$ is the one in the space $\wedge^{p} V^{\prime}$. With the above normalization, the constant function $d z_{1} \wedge \cdots \wedge d z_{p}$ has norm 1 in the weighted Bergman space. The computation of the normalization constant is done below.

When $p=n$, the space is the usual weighted Bergman space of scalar holomorphic functions $f(z)=f(z) d z_{1} \wedge \cdots \wedge d z_{n}$ so that

$$
\|f\|_{\nu}^{2}=\frac{1}{d_{\nu}} \int_{B^{n}}|f(z)|^{2}\left(1-|z|^{2}\right)^{\nu} d m(z)<\infty
$$

for $B(z, z)^{t} d z_{1} \wedge \cdots \wedge d z_{n}=\left(1-|z|^{2}\right)^{n+1} d z_{1} \wedge \cdots \wedge d z_{n}$.

The group $G$ acts on $\mathcal{A}_{\nu, p}$ via

$$
\pi_{\nu}(g) f(z)=J_{g^{-1}}(z)^{\frac{\nu}{n+1}}\left(d g^{-1}(z)\right)^{t} f\left(g^{-1} z\right),
$$


where $J_{g^{-1}}(z)$ stands for the complex Jacobian of $g^{-1}$ and $\left(d g^{-1}(z)\right)^{t}$ : $\wedge^{p} V^{\prime} \rightarrow \wedge^{p} V^{\prime}$ the pull-back of the differential $d g^{-1}(z): V \rightarrow V$. The reproducing kernel of the space $\mathcal{A}_{\nu, p}$ is

$$
(1-(z, w))^{-\nu} \otimes^{p}\left(B^{t}(z, w)\right)^{-1}
$$

this can easily be obtained by the transformation rule of the kernel under $G$. To find the expansion of the reproducing kernel we need to find the orthogonal decomposition of the space $\mathcal{A}_{\nu, p}$ under the compact group $K$. The space $\mathcal{P}\left(\wedge^{p} V^{\prime}\right)$ of all $\wedge^{p} V^{\prime}$-valued holomorphic polynomials is dense in the space $\mathcal{A}_{\nu, p}$, and as representation space of $K$ it is

$$
\mathcal{P} \otimes \wedge^{p} V^{\prime}=\sum_{m=0}^{\infty} \mathcal{P}_{m} \otimes \wedge^{p} V^{\prime} .
$$

We can further decompose it into irreducible representations of $K$. Let $q=n-p$. Recall that $\mathcal{P}_{m}$ has highest weight $-m \varepsilon_{n}$ and $\wedge^{p} V^{\prime}$ has highest weight $-\varepsilon_{q+1}-\cdots-\varepsilon_{n}$. Thus, if $m \geq 1, \mathcal{P}_{m} \otimes \wedge^{p} V^{\prime}$ is decomposed into a sum of two modules with highest weights $-m \varepsilon_{n}-\varepsilon_{q+1}-\cdots-\varepsilon_{n}$ and $-m \varepsilon_{n}-\varepsilon_{q}-\cdots-\varepsilon_{n-1}$; we denote the two modules by $V^{-m \varepsilon_{n}-\varepsilon_{q+1}-\cdots-\varepsilon_{n}}$ and $V^{-m \varepsilon_{n}-\varepsilon_{q}-\cdots-\varepsilon_{n-1}}$ respectively. That is

$$
\mathcal{P} \otimes \wedge^{p} V^{\prime}=\sum_{m=0}^{\infty} V^{-m \varepsilon_{n}-\varepsilon_{q+1}-\cdots-\varepsilon_{n}} \oplus V^{-m \varepsilon_{n}-\varepsilon_{q}-\cdots-\varepsilon_{n-1}},
$$

where it is understood that $V^{-m \varepsilon_{n}-\varepsilon_{q}-\cdots-\varepsilon_{n-1}}$ for $m=0$ will not appear. Note that for $p=n$ the second summand does not make sense and only the first summand appears.

This next result is similar to Lemma 1 in [7].

Lemma 3.1. The highest weight vectors of the above spaces are given by

$$
v_{-m \varepsilon_{n}-\varepsilon_{q+1}-\cdots-\varepsilon_{n}}=\sqrt{\frac{p !}{m !}} z_{n}^{m} d z_{q+1} \wedge \cdots \wedge d z_{n}
$$

and

$$
\begin{aligned}
& v_{-m \varepsilon_{n}-\varepsilon_{q}-\cdots-\varepsilon_{n-1}} \\
& =\sqrt{\frac{p !}{(m-1) !(m+p)} \sum_{j=q}^{n}(-1)^{n-j} z_{n}^{m-1} z_{j} d z_{q} \wedge \cdots \wedge{\widehat{d z_{j}}} \wedge \cdots \wedge d z_{n},}
\end{aligned}
$$

where $\widehat{d z}_{j}$ indicates that the term is missing in the wedge product. The two vectors are unit vectors with respect to the Fock norm.

Proof. The first formula is clear. We prove that the second vector is annihilated by the positive root vectors. Observe that, for $j<k$,

$$
E_{j, k} d z_{j}=-d z_{k}, E_{j, k} d z_{l}=0, l \neq j
$$


and

$$
E_{j, k} z_{n}^{m-1} z_{j}=-z_{n}^{m-1} z_{k}, E_{j, k} z_{n}^{m-1} z_{l}=0, l \neq j .
$$

Consider a positive root vector $E_{j_{0}, k_{0}}, j_{0}<k_{0}$. If $j_{0}<q$ it annihilates every term in the sum. Let $q \leq j_{0}<k_{0}$. If $k_{0} \leq n-1$ we have

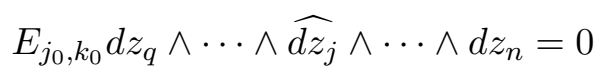

for $j \neq k_{0}$ and

$E_{j_{0}, k_{0}} d z_{q} \wedge \cdots \wedge \widehat{d z_{k_{0}}} \wedge \cdots \wedge d z_{n}=(-1) d z_{q} \wedge \cdots \wedge d z_{k_{0}} \wedge \cdots \wedge \widehat{d z_{k_{0}}} \wedge \cdots \wedge d z_{n}$ with $d z_{k_{0}}$ appearing in the $j_{0}$ th position. We rewrite it as $E_{j_{0}, k_{0}} d z_{q} \wedge \cdots \wedge \widehat{d z_{k_{0}}} \wedge \cdots \wedge d z_{n}=(-1)^{k_{0}-j_{0}} d z_{q} \wedge \cdots \wedge \widehat{d z_{j_{0}}} \wedge \cdots \wedge d z_{k_{0}} \wedge \cdots \wedge d z_{n}$ by moving $d z_{k_{0}}$ to the $k_{0}$ th (empty) position. Using (3.2) and (3.3) we see that $E_{j_{0}, k_{0}}$ acting on $v_{-m \varepsilon_{n}-\varepsilon_{q}-\cdots-\varepsilon_{n-1}}$ is, disregarding the normalization constant factor,

$$
\begin{aligned}
& -(-1)^{n-j_{0}} z_{n}^{m-1} z_{k_{0}} d z_{q} \wedge \cdots \wedge \widehat{d z_{j_{0}}} \wedge \cdots \wedge d z_{n} \\
& \quad+(-1)^{n-k_{0}}(-1)^{k_{0}-j_{0}} z_{n}^{m-1} z_{k_{0}} d z_{q} \wedge \cdots \wedge \widehat{d z_{j_{0}}} \wedge \cdots \wedge d z_{n},
\end{aligned}
$$

which is clearly 0 . The case when $k_{0}=n$ can be proved similarly.

For any highest weight $m_{1} \varepsilon_{1}+\cdots+m_{n} \varepsilon_{n}$ appearing in the space $\mathcal{P}\left(V^{\prime}\right)$ we let $K_{m_{1} \varepsilon_{1}+\cdots+m_{n} \varepsilon_{n}}(z, w)$ be the reproducing kernel of the space with respect to the Fock norm. To find the expansion of the reproducing kernel in terms of $K_{m_{1} \varepsilon_{1}+\cdots+m_{n} \varepsilon_{n}}$ we need to calculate the norm of the highest weight vector in the space.

The computation of the norms of the highest weight vectors in the case $p=1$ differs somewhat from the case $p>1$. We start with $p=1$.

Lemma 3.2. The norm of $v_{-m \varepsilon_{n}-\varepsilon_{n}}$ and $v_{-m \varepsilon_{n}-\varepsilon_{n-1}}$ in the weighted Bergman space is given by the following:

$$
\left\|v_{-m \varepsilon_{n}-\varepsilon_{n}}\right\|_{\nu}^{2}=\frac{1}{(\nu+2)_{m}}, \quad\left\|v_{-m \varepsilon_{n}-\varepsilon_{n-1}}\right\|_{\nu}^{2}=\frac{\nu+1}{(\nu)_{m+1}} .
$$

Proof. Consider first the vector $v=v_{-m \varepsilon_{n}-\varepsilon_{n}}$. We have

$$
\left(B(z, z)^{t} v, v\right)=(v B(z, z), v)=\frac{1}{m !}\left(1-|z|^{2}\right)\left(\left|z_{n}^{m}\right|^{2}-\left|z_{n}^{m+1}\right|^{2}\right) .
$$

The norm then becomes, disregarding temporarily the normalizing constant and $\frac{1}{m !}$,

$$
\int_{B}\left(\left|z_{n}^{m}\right|^{2}-\left|z_{n}^{m+1}\right|^{2}\right)\left(1-|z|^{2}\right)^{\nu+1} \frac{d m(z)}{\left(1-|z|^{2}\right)^{n+1}} .
$$

Recalling that (see e.g., [9], Prop. 1.4.9)

$$
\int_{B}\left|z_{1}^{k_{1}} \ldots z_{n}^{k_{n}}\right|\left(1-|z|^{2}\right)^{\nu} \frac{d m(z)}{\left(1-|z|^{2}\right)^{n+1}}=C_{\nu} \frac{k_{1} ! \ldots k_{n} !}{(\nu)_{k_{1}+\cdots+k_{n}}}
$$


with

$$
C_{\nu}=\pi^{n} \frac{\Gamma(\nu-n)}{\Gamma(\nu)}
$$

we find the above integral is

$$
C_{\nu+1}\left(\frac{m !}{(\nu+1)_{m}}-\frac{(m+1) !}{(\nu+1)_{m+1}}\right)=C_{\nu+1} \frac{m !}{(\nu+1)_{m}} \frac{\nu}{\nu+m+1} .
$$

Putting these calculations together we find

$$
\|v\|_{\nu}^{2}=\left\|v_{-m \varepsilon_{n}-\varepsilon_{n}}\right\|_{\nu}^{2}=\frac{1}{(\nu+2)_{m}} .
$$

Now let $v=v_{-m \varepsilon_{n}-\varepsilon_{n-1}}=\frac{1}{\sqrt{(m-1) !(m+1)}} w$.

$$
\begin{aligned}
B(z, z)^{t} w & =\left(z_{n}^{m} e_{n-1}^{\prime}-z_{n}^{m-1} z_{n-1} e_{n}^{\prime}\right) B(z, z) \\
& =\left(1-|z|^{2}\right)\left(z_{n}^{m} e_{n-1}^{\prime}-z_{n}^{m-1} z_{n-1} e_{n}^{\prime}\right)\left(I-z \bar{z}^{\prime}\right) \\
& =\left(1-|z|^{2}\right)\left(z_{n}^{m} e_{n-1}^{\prime}-z_{n}^{m} z_{n-1} \bar{z}^{\prime}-z_{n}^{m-1} z_{n-1} e_{n}^{\prime}+z_{n}^{m} z_{n-1} \bar{z}^{\prime}\right) \\
& =\left(1-|z|^{2}\right)\left(z_{n}^{m} e_{n-1}^{\prime}-z_{n}^{m-1} z_{n-1} e_{n}^{\prime}\right)
\end{aligned}
$$

which is remarkably simple. Thus

$$
(B(z, z) w, w)=\left|z_{n}^{m}\right|^{2}+\left|z_{n}^{m-1} z_{n-1}\right|^{2} .
$$

The rest is straightforward computation.

Consequently, we have:

Theorem 3.3. The reproducing kernel $\left(B^{t}(z, w)\right)^{-1}(1-(z, w))^{-\nu}$ has the following expansion:

$$
\begin{aligned}
& \left(B^{t}(z, w)\right)^{-1}(1-(z, w))^{-\nu} \\
& =I+\sum_{m=1}^{\infty}\left[(\nu+2)_{m} K_{-m \varepsilon_{n}-\varepsilon_{n}}(z, w)+\frac{(\nu)_{m+1}}{\nu+1} K_{-m \varepsilon_{n}-\varepsilon_{n-1}}(z, w)\right] .
\end{aligned}
$$

We can then determine the composition series by reading off the order of the poles of the $\mathfrak{g}$-invariant Hermitian structure on $\mathcal{P} \otimes V^{\prime}$ for all parameters $\nu$.

Theorem 3.4. The representation $\mathcal{P} \otimes V^{\prime}$ of $\mathfrak{g}$ is reducible if and only if $\nu=0$ or $\nu \leq-2$ is an integer, in which case we have a composition series

$$
0=M_{0} \subset M_{1} \subset M_{2}=\mathcal{P} \otimes V^{\prime},
$$

and the only unitarizable quotient is $M_{1} / M_{0}=M_{1}$ when $\nu=0$. The K-types in composition factors are described as follows:

$$
M_{1}=V^{\prime}+\sum_{m \geq 0} V^{-m \varepsilon_{n}-\varepsilon_{n}}
$$


if $\nu=0$;

$$
M_{1}=V^{\prime}+V^{-\varepsilon_{n}-\varepsilon_{n-1}}
$$

if $\nu=-2$; and

$$
M_{1}=V^{\prime}+\sum_{1 \leq m \leq-(\nu+1)} V^{-m \varepsilon_{n}-\varepsilon_{n}}+\sum_{1 \leq m \leq-\nu} V^{-m \varepsilon_{n}-\varepsilon_{n-1}},
$$

if $\nu \leq-3$.

Remark 3.5. When $\nu=0$, the expansion becomes

$$
\left(B^{t}(z, w)\right)^{-1}=\sum_{m=0}^{\infty}(\nu+2)_{m} K_{-m \varepsilon_{n}-\varepsilon_{n}}(z, w)
$$

the corresponding unitary representation $M_{1}$ consists of $K$-types $-m \varepsilon_{n}-\varepsilon_{n}$, $m=0,1, \ldots$, and it realizes the so-called Dirichlet space. Consider the analytic continuation of the scalar holomorphic discrete series with reproducing kernel $(1-(z, w))^{-\kappa}$ with the corresponding action of $G$. When $\kappa=0$, the space $\mathcal{P}$ of all polynomials is reducible and the space $\mathbb{C}$ of constant function is a submodule, considered as representations of $\mathfrak{g}^{\mathbb{C}}$. The quotient $\mathcal{P} / \mathbb{C}$ is also unitarizable and is equivalent to our module $M_{1}$ with $\nu=0$; so the quotient is no longer a continuation of the scalar discrete series. The intertwining operator from the quotient $\mathcal{P} / \mathbb{C}$ onto $M_{1}$ is $f \mapsto \partial f$. We remark that Theorem 5.4 in [3] gives the equivalence of the top quotient of a composition series at the reducible point in analytic continuation of a scalar discrete series, with another highest weight module of the same series. However, the theorem, as it stands there, holds only for tube domains. For non-tube domains the quotients there is equivalent to an analytic continuation of the vector-valued discrete series, since the highest weight given in that theorem is not one-dimensional; the case of the unit ball is as explained here. One of the authors (GZ) pointed this out to Professor Jacques Faraut in Mittag-Leffler institute in May 1996, who immediately realized that the theorem should be modified for non-tube domains.

Remark 3.6. When $\nu=-2$, the expansion reads as follows:

$$
\left(B^{t}(z, z)\right)^{-1}\left(1-|z|^{2}\right)^{2}=I-2 K_{-\varepsilon_{n}-\varepsilon_{n-1}}(z, z) .
$$

The representation $-\varepsilon_{n}-\varepsilon_{n-1}$ is the space of anti-symmetric tensor of $V^{\prime}$ with $V^{\prime}$, and as polynomials it has orthogonal basis $z_{i} e_{j}^{\prime}-z_{j} e_{i}^{\prime}, i<j$. One can easily compute the reproducing kernel and finds that

$$
K_{-\varepsilon_{n}-\varepsilon_{n-1}}=\frac{1}{2}\left(|z|^{2}-z \otimes z^{*}\right)^{t} .
$$

On the other hand, by $(2.1)$ we have

$$
\left(B^{t}(z, z)\right)^{-1}\left(1-|z|^{2}\right)^{2}=\left(1-|z|^{2}+z \otimes z^{*}\right)^{\prime}=1-\left(|z|^{2}+z \otimes z^{*}\right)^{t},
$$

which clearly coincides with (3.5) and (3.6). 
Consider now $p>1$. Let $v=v_{-m \varepsilon_{n}-\varepsilon_{q+1}-\cdots-\varepsilon_{n}}$ and write $v=\sqrt{\frac{p !}{m !}} w$. We first compute $B(z, z) w$,

$$
\begin{aligned}
& \otimes^{p} B(z, z)^{t} w \\
& =\left(1-|z|^{2}\right)^{p} z_{n}^{m}\left(d z_{q+1} \wedge \cdots \wedge d z_{n}-\sum_{j=q+1}^{n} d z_{q+1} \wedge \cdots \wedge z_{j} z^{*} \wedge \cdots \wedge d z_{n}\right),
\end{aligned}
$$

where in the summation the term $z_{j} z^{*}$ appears in the $j$ th position. Indeed,

$$
\begin{aligned}
& \otimes^{p} B(z, z)^{t} d z_{q+1} \wedge \cdots \wedge d z_{n} \\
& =\left(1-|z|^{2}\right)^{p}\left(d z_{q+1}-z_{q+1} z^{*}\right) \wedge \cdots \wedge\left(d z_{n}-z_{n} z^{*}\right) \\
& =\left(1-|z|^{2}\right)^{p}\left(d z_{q+1} \wedge \cdots \wedge d z_{n}-\sum_{j=q+1}^{n} d z_{q+1} \wedge \cdots \wedge z_{j} z^{*} \wedge \cdots \wedge d z_{n}\right)
\end{aligned}
$$

since all other wedge products contain two factor of $z^{*}$ and are vanishing. Consequently

$$
\begin{aligned}
& \left(\otimes^{p} B(z, z)^{t} w, w\right) \\
& =\left(1-|z|^{2}\right)^{p} z_{n}^{m}\left(d z_{q+1} \wedge \cdots \wedge d z_{n}-\sum_{j=q+1}^{n} d z_{q+1} \wedge \cdots \wedge z_{j} z^{*} \wedge \cdots \wedge d z_{n}, w\right) \\
& =\frac{1}{p !}\left(1-|z|^{2}\right)^{p}\left|z_{n}\right|^{2 m}\left(1-\sum_{j=q+1}^{n}\left|z_{j}\right|^{2}\right) .
\end{aligned}
$$

We can compute its integration, noticing that the integral involving $\left|z_{n}\right|^{2 m}\left|z_{j}\right|^{2}$ for $j=n$ is different from that for $j \neq n$,

$$
\begin{aligned}
\|v\|_{\nu}^{2}= & \frac{1}{m !} \frac{1}{d_{\nu}} \int_{B}\left(1-|z|^{2}\right)^{p}\left(\left|z_{n}\right|^{2 m}\right. \\
& \left.-\sum_{j=q+1}^{n-1}\left|z_{n}\right|^{2 m}\left|z_{j}\right|^{2}-\left|z_{n}\right|^{2 m}\left|z_{n}\right|^{2}\right)\left(1-|z|^{2}\right)^{\nu-n-1} d m(z) \\
= & \frac{1}{m !} \frac{1}{d_{\nu}} \frac{\pi^{n} \Gamma(\nu+p-n)}{\Gamma(\nu+p)}\left(\frac{m !}{(\nu+p)_{m}}\right. \\
& \left.-(p-1) \frac{m !}{(\nu+p)_{m+1}}-\frac{(m+1) !}{(\nu+p)_{m+1}}\right) \\
= & \frac{1}{d_{\nu}} \frac{\pi^{n} \Gamma(\nu+p-n)}{\Gamma(\nu+p)} \frac{1}{(\nu+p)_{m}}\left(1-\frac{p-1}{\nu+p+m}-\frac{m+1}{\nu+p+m}\right) \\
= & \frac{1}{d_{\nu}} \frac{\pi^{n} \Gamma(\nu+p-n)}{\Gamma(\nu+p)} \frac{1}{(\nu+p)_{m}} \frac{\nu+p+m}{\nu+p} \\
= & \frac{1}{(\nu+p+1)_{m}} .
\end{aligned}
$$


Next let $v=v_{-m \varepsilon_{n}-\varepsilon_{q}-\cdots-\varepsilon_{n-1}}$ and write $v=\sqrt{\frac{p !}{(m-1) !(m+p)}} w$. We have

$$
\left(B(z, z)^{t} w, w\right)=\left(1-|z|^{2}\right)^{p}\left(\otimes^{p}\left(1-z \otimes z^{*}\right)^{t} w, w\right) .
$$

However,

$$
\begin{aligned}
& \otimes^{p}\left(1-z \otimes z^{*}\right)^{t} w \\
& =\sum_{j=q}^{n}(-1)^{n-j} z_{n}^{m-1} z_{j}\left(d z_{q}-z_{q} z^{*}\right) \wedge \cdots \wedge \widehat{d z_{j}} \wedge \cdots \wedge\left(d z_{n}-z_{n} z^{*}\right) \\
& =w-\sum_{j=q}^{n} \sum_{i \neq j, i=q}^{n}(-1)^{n-j} z_{n}^{m-1} z_{j} d z_{q} \wedge \cdots \wedge z_{i} z^{*} \wedge \cdots \wedge \widehat{d z}_{j} \wedge \cdots \wedge d z_{n}
\end{aligned}
$$

so that

$$
\begin{aligned}
& \left(\otimes^{p}\left(1-z \otimes z^{*}\right)^{t} w, w\right) \\
& =(w, w)-\sum_{j=q}^{n} \sum_{i \neq j, i=q}^{n}(-1)^{n-j} z_{n}^{m-1} z_{j} z_{i} \\
& \quad \cdot\left(d z_{q} \wedge \cdots \wedge z^{*} \wedge \cdots \wedge \widehat{d z_{j}} \wedge \cdots \wedge d z_{n}, w\right) .
\end{aligned}
$$

We prove that the second double sum actually vanishes. Indeed writing $z^{*}=\bar{z}_{1} d z_{1}+\cdots+\bar{z}_{n} d z_{n}$, we see that only the term $\bar{z}_{i} d z_{i}+\bar{z}_{j} d z_{j}$ contributes to the wedge product and then the inner product, namely

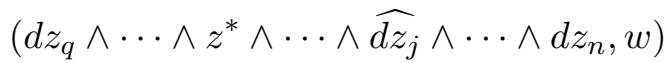

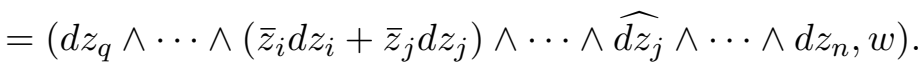

Furthermore

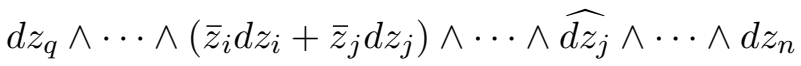

$$
\begin{aligned}
& =\bar{z}_{i} d z_{q} \wedge \cdots \wedge d z_{i} \wedge \cdots \wedge{\widehat{d z_{j}}} \wedge \cdots \wedge d z_{n} \\
& +\bar{z}_{j} d z_{q} \wedge \cdots \wedge d z_{j} \wedge \cdots \wedge{\widehat{d z_{j}}} \wedge \cdots \wedge d z_{n} \\
& =\bar{z}_{i} d z_{q} \wedge \cdots \wedge d z_{i} \wedge \cdots \wedge{\widehat{d z_{j}}} \wedge \cdots \wedge d z_{n}
\end{aligned}
$$

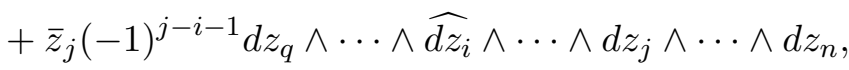

where in the last term we move $d z_{j}$ in the $i$ th position to the $j$ th empty position (leaving the $i$ th position empty). The double sum is then 


$$
\begin{aligned}
& \sum_{j=q}^{n} \sum_{i \neq j, i=q}^{n}(-1)^{n-j} z_{n}^{m-1} z_{j}\left|z_{i}\right|^{2}\left(d z_{q} \wedge \cdots \wedge d z_{i} \wedge \cdots \wedge \widehat{d z_{j}} \wedge \cdots \wedge d z_{n}, w\right) \\
& \quad+\sum_{j=q}^{n} \sum_{i \neq j, i=q}^{n} z_{i} \bar{z}_{j}(-1)^{j-i-1}\left(d z_{q} \wedge \cdots \wedge \widehat{d z_{i}} \wedge \cdots \wedge d z_{j} \wedge \cdots \wedge d z_{n}, w\right) \\
& =\sum_{j=q}^{n} \sum_{i \neq j, i=q}^{n}\left|(-1)^{n-j} z_{n}^{m-1} z_{j}\right|^{2}\left|z_{i}\right|^{2} \frac{1}{p !} \\
& \quad+\sum_{j=q}^{n} \sum_{i \neq j, i=q}^{n}(-1)^{n-j}(-1)^{n-i}(-1)^{j-i-1} z_{n}^{m-1} z_{j} z_{i} \bar{z}_{j} \frac{z_{n}^{m-1} z_{i}}{p !} \\
& =0 .
\end{aligned}
$$

So that only the first term contributes to the integral, and

$$
\begin{aligned}
\|w\|_{\nu}^{2} & =\frac{1}{d_{\nu}} \int_{B}(w, w)\left(1-|z|^{2}\right)^{p+\nu-n-1} d m(z) \\
& =\frac{1}{d_{\nu}} \frac{1}{p !} \int_{B} \sum_{j=q}^{n}\left|z_{n}^{m-1} z_{j}\right|^{2} \mid\left(1-|z|^{2}\right)^{p+\nu-n-1} d m(z) \\
& =\frac{1}{d_{\nu}} \frac{1}{p !} \frac{\pi^{n} \Gamma(p+\nu-n)}{\pi^{n} \Gamma(p+\nu)}\left((n-q) \frac{(m-1) !}{(\nu+p)_{m}}+\frac{m !}{(\nu+p)_{m}}\right) \\
& =\frac{1}{d_{\nu}} \frac{1}{p !} \frac{\pi^{n} \Gamma(p+\nu-n)}{\pi^{n} \Gamma(p+\nu)} \frac{(m-1) !}{(\nu+p)_{m}}(n-q+m) .
\end{aligned}
$$

We find eventually

$$
\|w\|_{\nu}^{2}=\frac{1}{\nu(\nu+p+1)_{m-1}} .
$$

We have thus established the following expansion; the case $p=n$ reduces to the known expansion for the weighted Bergman reproducing kernel (1$(z, w))^{-\nu+n+1}$ :

Theorem 3.7. Let $1<p \leq n$. The reproducing kernel $\otimes^{p}\left(B^{t}(z, w)\right)^{-1}(1-$ $(z, w))^{-\nu}$ has the following expansion:

$$
\begin{aligned}
& \otimes^{p}\left(B^{t}(z, w)\right)^{-1}(1-(z, w))^{-\nu} \\
& =I+\sum_{m=1}^{\infty}\left[(\nu+p+1)_{m} K_{-m \varepsilon_{n}-\varepsilon_{q+1}-\cdots-\varepsilon_{n}}(z, w)\right. \\
& \left.\quad+\nu(\nu+p+1)_{m-1} K_{-m \varepsilon_{n}-\varepsilon_{q}-\ldots \varepsilon_{n-1}}(z, w)\right]
\end{aligned}
$$

where $q=n-p$.

Similar to Theorem 3.4 we have: 
Theorem 3.8. Let $1<p \leq n$. The representation $\mathcal{P} \otimes \wedge^{p} V^{\prime}$ is reducible if and only if $\nu=0$ or $\nu \leq-(p+1)$ is an integer, in which case the composition factors $0=M_{0} \subset M_{1} \subset M_{2}=\mathcal{P} \otimes \wedge^{p} V^{\prime}$ are described as follows:

$$
M_{1}=\sum_{m=0}^{\infty} V^{-m \varepsilon_{n}-\varepsilon_{q+1}-\cdots-\varepsilon_{n}}
$$

if $\nu=0$;

$$
M_{1}=V^{-\varepsilon_{q+1}-\cdots-\varepsilon_{n}}+V^{-\varepsilon_{n}-\varepsilon_{q}-\cdots-\varepsilon_{n}}
$$

if $\nu=-(p+1)$; and

$$
M_{1}=\sum_{0 \leq m \leq-\nu-p-1} V^{-m \varepsilon_{n}-\varepsilon_{q+1}-\cdots-\varepsilon_{n}}+\sum_{1 \leq m \leq-\nu-p} V^{-m \varepsilon_{n}-\varepsilon_{q}-\cdots-\varepsilon_{n}}
$$

if $\nu<-(p+1)$.

It is interesting to observe that when $\nu=0$ we have

$$
\otimes^{p}\left(B^{t}(z, w)\right)^{-1}=\sum_{m=0}^{\infty}(\nu+p+1)_{m} K_{-m \varepsilon_{n}-\varepsilon_{q+1}-\cdots-\varepsilon_{n}}(z, w)
$$

so that there exists a unitarizable submodule of $\mathcal{P}\left(\wedge^{p} V^{\prime}\right)$ consisting of the $K$-types $-m \varepsilon_{n}-\varepsilon_{q+1}-\cdots-\varepsilon_{n}$, for all $p=1, \ldots, n$; only when $p=n$ is that a discrete series, namely the Bergman space.

\section{Hilbert spaces of holomorphic vector-fields and antisymmetric tensor fields.}

Let $1 \leq q \leq n$. In this section we study the space $\mathcal{B}_{\nu, q}=L_{a}^{2}\left(B^{n}, \wedge^{q} V, \mu_{\nu}\right)$, $\nu>2 q+n$, of $\wedge^{q} V$-valued holomorphic functions $f$ on $B^{n}$ so that

$$
\|f\|_{\nu}^{2}=\frac{1}{b_{\nu}} \int_{B^{n}}\left(\wedge^{q} B(z, z)^{-1} f(z), f(z)\right) d \mu_{\nu}(z)<\infty
$$

and its analytic continuation in $\nu$. Here

$$
b_{\nu}=\pi^{n} \frac{\Gamma(\nu-q-1-n)(\nu-1-n)}{\Gamma(\nu-q)} .
$$

Notice that if $q=n$ the space $\wedge^{n} V$ is one dimensional and the above space is the weighted Bergman space of holomorphic functions $f(z)=f(z) e_{1} \wedge \cdots \wedge e_{n}$ so that

$$
\|f\|_{\nu}^{2}=\frac{1}{b_{\nu}} \int_{B^{n}}|f(z)|^{2}\left(1-|z|^{2}\right)^{\nu-2 n-2} d m(z)<\infty .
$$

The group $G$ acts on $\mathcal{B}_{\nu, q}$ via

$$
\tau_{\nu} f(z)=J_{g^{-1}}(z)^{\frac{\nu}{n+1}}\left(d g^{-1}(z)\right)^{-1} f\left(g^{-1} z\right) .
$$

The reproducing kernel of $\mathcal{B}_{\nu, q}$ is then

$$
(1-(z, w))^{-\nu} \wedge^{q} B(z, w) .
$$


Remark 4.1. Let $q=1$. The space $L_{a}^{2}\left(B^{n}, V, \mu_{\nu}\right)$ appears also naturally in the study of discrete spectrum of Laplace-Beltrami operators on line bundles over the unit ball. Indeed consider the $L^{2}$-space $L^{2}\left(B^{n}, \mu_{\nu}\right)$ on $B^{n}$ with respect to the weighted measure $\mu_{\nu}$. The group $G$ acts unitarily on the space and there is an $G$-invariant (nonpositive) Laplace-Beltrami operator. It has some discrete spectrum, if $\nu>n+1$, the biggest discrete spectrum corresponds to the weighted Bergman space $L_{a}^{2}\left(B^{n}, \mu_{\nu}\right)$; if $\nu>n+2$, the next one, say $A_{1}^{\nu}\left(B^{n}\right)$ gives an invariant subspace consisting of non-holomorphic functions, see [14]. The invariant Cauchy-Riemann operator $\bar{D}$ is the unitary intertwining operator from $A_{1}^{\nu}\left(B^{n}\right)$ onto $\mathcal{B}_{\nu, q}=L_{a}^{2}\left(B^{n}, V, \mu_{\nu}\right)$; see [8] and $[13]$.

The space of $\mathcal{P}\left(\wedge^{q} V\right)$ all $\wedge^{q} V$-valued holomorphic polynomials is

$$
\mathcal{P}\left(\wedge^{q} V\right)=\mathcal{P} \otimes \wedge^{q} V=\sum_{m=0}^{\infty} \mathcal{P}_{m} \otimes \wedge^{q} V .
$$

As a representation of $K$ the space $\wedge^{q} V$ is an irreducible representation with highest weight $\varepsilon_{1}+\cdots+\varepsilon_{q}$. Thus the polynomial space is decomposed further as

$$
\mathcal{P} \otimes \wedge^{q} V=V^{\varepsilon_{1}+\cdots+\varepsilon_{q}}+\sum_{m=1}^{\infty}\left(V^{-m \varepsilon_{n}+\varepsilon_{1}+\cdots+\varepsilon_{q}}+V^{-m \varepsilon_{n}+\varepsilon_{1}+\cdots+\varepsilon_{q-1}+\varepsilon_{n}}\right),
$$

where it is understood that $V^{-m \varepsilon_{n}+\varepsilon_{1}+\cdots+\varepsilon_{q-1}+\varepsilon_{n}}$ for $m=0$ will not appear.

We give now formulas for the highest weight vectors of the spaces above. For $q=1$ they are proved in Lemma 1, [7], the general case is similar and we omit the proof.

Lemma 4.2. We have the following formulas for the highest weigh vectors in the respective spaces:

$$
v_{-m \varepsilon_{n}+\varepsilon_{1}+\cdots+\varepsilon_{q}}=\sqrt{\frac{q !}{m !}} z_{n}^{m} e_{1} \wedge \cdots \wedge e_{q}
$$

and respectively

$v_{-m \varepsilon_{n}+\varepsilon_{1}+\cdots+\varepsilon_{q-1}+\varepsilon_{n}}=\frac{\sqrt{q !}}{\sqrt{(m-1) !(m+n-q)}} \sum_{j=q}^{n} z_{n}^{m-1} z_{j} e_{1} \wedge \cdots \wedge e_{q-1} \wedge e_{j}$.

The two vectors are unit vectors with respect to the Fock norm.

We now compute the norm of the highest weight vectors in the weighted Bergman space. Let $q=1$. Notice that

$$
\begin{aligned}
& \left(B(z, z)^{-1} v_{-m \varepsilon_{n}+\varepsilon_{1}}, v_{-m \varepsilon_{n}+\varepsilon_{1}}\right) \\
& =\frac{1}{m !}\left(\left(1-|z|^{2}\right)^{-1}\left|z_{n}^{m}\right|^{2}+\left(1-|z|^{2}\right)^{-2}\left|z_{1}\right|^{2}\left|z_{n}^{m}\right|^{2}\right),
\end{aligned}
$$


its integral against $d \mu_{\nu}$ is

$$
\frac{1}{m !} b_{\nu}\left(C_{\nu-1} \frac{m !}{(\nu-1)_{m}}+C_{\nu-2} \frac{(m+1) !}{(\nu-2)_{m+1}} \frac{m !}{(m+1) !}\right)=\frac{1}{(\nu-1)_{m}} .
$$

Similarly,

$$
\begin{aligned}
& \left(B(z, z)^{-1} v_{-m \varepsilon_{n}+\varepsilon_{n}}, v_{-m \varepsilon_{n}+\varepsilon_{n}}\right) \\
& \left.=\frac{1}{(m-1) !(m+n+1)}\left(1-|z|^{2}\right)^{-1}|z|^{2}\left|z_{n}^{m-1}\right|^{2}+\left(1-|z|^{2}\right)^{-2}|z|^{4}\left|z_{n}^{m-1}\right|^{2}\right) .
\end{aligned}
$$

To calculate its integral against the measure $d \mu_{\nu}$ we write $|z|^{2}=1-\left(1-|z|^{2}\right)$, the above is then

$$
\frac{1}{(m-1) !(m+n+1)}\left(\left|z_{n}^{m-1}\right|^{2}\left(1-|z|^{2}\right)^{-2}-\left|z_{n}^{m-1}\right|^{2}\left(1-|z|^{2}\right)^{-1}\right) .
$$

We calculate the integral of (4.2) of the term in the parenthesis, and find

$$
\begin{aligned}
& b_{\nu}^{-1} C_{\nu-2} \frac{(m-1) !}{(\nu-2)_{m-1}}-b_{\nu}^{-1} C_{\nu-1} \frac{(m-1) !}{(\nu-1)_{m-1}} \\
& =\frac{\Gamma(\nu-1)}{\nu-1-n} \frac{(m-1) !(m+n-1)}{\Gamma(\nu+m-2)} .
\end{aligned}
$$

Thus the norm of $v_{-m \varepsilon_{n}+\varepsilon_{n}}$ is

$$
\left\|v_{-m \varepsilon_{n}+\varepsilon_{n}}\right\|_{\nu}^{2}=\frac{\Gamma(\nu-1)}{\nu-1-n} \frac{1}{\Gamma(\nu+m-2)}=\frac{1}{\left.(\nu-1-n)(\nu-1)_{m-1}\right)} .
$$

We have the following expansion for the reproducing kernel:

Theorem 4.3. The reproducing kernel $B(z, w)(1-(z, w))^{-\nu}$ has the following expansion:

$$
\begin{aligned}
& B(z, w)(1-(z, w))^{-\nu} \\
& =I+\sum_{m=1}^{\infty}\left[(\nu-1)_{m} K_{-m \varepsilon_{n}+\varepsilon_{1}}+(\nu-1-n)(\nu-1)_{m-1} K_{-m \varepsilon_{n}+\varepsilon_{n}}\right] .
\end{aligned}
$$

Theorem 4.4. The representation $\mathcal{P} \otimes V$ of $\mathfrak{g}^{\mathbb{C}}$ is reducible if and only if when $\nu=n+1$ or $\nu \leq 1$ is an integer; it has a composition series

$$
0=M_{0} \subset M_{1} \subset M_{2}=\mathcal{P} \otimes V ;
$$

the composition factor are given as follows:

$$
M_{1}=\sum_{m=0}^{\infty} V^{-m \varepsilon_{n}+\varepsilon_{1}},
$$


if $\nu=n+1$, and the composition factor are given as follows:

$$
M_{1}=V^{\varepsilon_{1}}+\sum_{1 \leq m<2-\nu} V^{-m \varepsilon_{n}+\varepsilon_{1}}+\sum_{1 \leq m<3-\nu} V^{-m \varepsilon_{n}+\varepsilon_{n}} ;
$$

the submodule $M_{1}$ is unitarizable if and only if $\nu=n+1$ and no quotient $M_{2} / M_{1}$ of the composition series is unitarizable.

Remark 4.5. We mention here that in the analytic continuation for the scalar holomorphic discrete series, the composition series has two factors at the reducible points, and the quotient is always unitarizable. Thus we see here that the unitarizable property behaves differently in the vector case.

Corollary 4.6. The Bergman operator has the following expansion:

$$
B(z, z)=I-K_{-\varepsilon_{n}+\varepsilon_{1}}-(1+n) K_{0}+(1+n) K_{-2 \varepsilon_{n}+\varepsilon_{n}}
$$

where

$$
K_{-\varepsilon_{n}+\varepsilon_{1}}=|z|^{2}-\frac{1}{n} z \otimes z^{*}, K_{0}=\frac{1}{n} z \otimes z^{*}, K_{-2 \varepsilon_{n}+\varepsilon_{n}}=\frac{1}{n+1}\left|z^{2}\right| z \otimes z^{*} .
$$

Remark 4.7. The above formula has the following nice explanation: Consider the space of $V$-valued polynomial space

$$
M_{1}=V^{\varepsilon_{1}} \oplus V^{-\varepsilon_{n}+\varepsilon_{1}} \oplus V^{0} \oplus V^{-2 \varepsilon_{n}+\varepsilon_{n}} .
$$

Under the $G$-action

$$
g f(z)=g^{\prime}(z)^{-1} f(g \cdot z)
$$

where $g \in G$ and $f$ is in the polynomial space, the space has an invariant Hermitian (non-unitary) form for which $B(z, z)$ is the reproducing kernel. Corollary 4.6 gives an expansion of $B(z, z)$ in terms of the reproducing kernels in each irreducible $K$-spaces. Actually $M_{1}$ is $G$-equivalent to the Lie algebra $\mathfrak{g}^{\mathbb{C}}$ with the adjoint action of $G$; and the above decomposition of $M_{1}$ is simply the decomposition of $\mathfrak{g}^{\mathbb{C}}$

$$
\mathfrak{g}^{\mathbb{C}}=\mathfrak{p}^{+} \oplus \mathfrak{k}_{s}^{\mathbb{C}} \oplus \mathfrak{z} \oplus \mathfrak{p}^{-}
$$

with respect to the action of $K$. Here $\mathfrak{z}$ is the center of $\mathfrak{k}^{\mathbb{C}}$, which is onedimensional. Clearly this can be done for all the bounded symmetric domain, we have thus given a different property of the Bergman operator.

We consider now the case $q>1$. Let $v=v_{-m \varepsilon_{n}+\varepsilon_{1}+\cdots+\varepsilon_{q}}$. Using (2.1) and by similar consideration as in $\S 3$ we have

$$
\left(\otimes^{q} B(z, z)^{-1} v, v\right)=\frac{1}{m !}\left(1-|z|^{2}\right)^{-q-1}\left|z_{n}^{m}\right|^{2}\left(1-|z|^{2}+\sum_{j=1}^{q}\left|z_{j}\right|^{2}\right)
$$


It is now easy to compute $\|v\|_{\nu}^{2}$. If $1<q<n,\|v\|_{\nu}^{2}$ is, disregarding the normalization constant,

$$
\begin{aligned}
& \frac{1}{m !} \int_{B}\left(1-|z|^{2}\right)^{-2 q}\left|z_{n}^{m}\right|^{2}\left(\left(1-|z|^{2}\right)^{q}\right. \\
& \left.\quad+\left(1-|z|^{2}\right)^{q-1} \sum_{j 1}^{q}\left|z_{j}\right|^{2}\right)\left(1-|z|^{2}\right)^{\nu-n-1} d m(z) \\
& =\frac{1}{m !}\left(\frac{\pi^{n} \Gamma(\nu-q-n)}{\Gamma(\nu-q)} \frac{m !}{(\nu-q)_{m}}+q \frac{\pi^{n} \Gamma(\nu-q-1-n)}{\Gamma(\nu-q-1)} \frac{m !}{(\nu-q-1)_{m+1}}\right) \\
& =\frac{\pi^{n} \Gamma(\nu-q-1-n)}{\Gamma(\nu-q+m)}(\nu-n),
\end{aligned}
$$

and that

$$
\|v\|_{\nu}^{2}=\frac{1}{(\nu-q)_{m}} .
$$

If $q=n$, Formula (4.4) becomes

$$
\left(\otimes^{q} B(z, z)^{-1} v, v\right)=\frac{1}{m !}\left(1-|z|^{2}\right)^{-q-1}\left|z_{n}^{m}\right|^{2}
$$

and

$$
\|v\|_{\nu}^{2}=\frac{1}{(\nu-n-1)_{m}},
$$

which is the known formula for the norm of $\frac{z_{n}^{m}}{\sqrt{m !}}$ in the scalar weighted Bergman space with weight $\left(1-|z|^{2}\right)^{\nu-2 n-2}$.

Finally consider $v=v_{-m \varepsilon_{n}+\varepsilon_{1}+\cdots+\varepsilon_{q-1}+\varepsilon_{n}}$. Write $w=\sum_{j=q}^{n} z_{j} e_{1} \wedge \cdots \wedge$ $e_{q-1} \wedge e_{j}$.

$$
\begin{aligned}
B(z, z)^{-1} w= & \left(1-|z|^{2}\right)^{-2 q} \otimes^{q}\left(1-|z|^{2}+z \otimes z^{*}\right) w \\
= & \left(1-|z|^{2}\right)^{-q} z_{n}^{m-1} w \\
& +\sum_{j=q}^{n} z_{j}\left(\sum_{i=1}^{q-1} e_{1} \wedge \cdots \wedge \overline{z_{i}} z \wedge \cdots \wedge e_{j}+e_{1} \wedge \cdots \wedge e_{q-1} \wedge \overline{z_{j}} z\right)
\end{aligned}
$$

where in the summation $\sum_{i=1}^{q-1}$ the factor $\overline{z_{i}} z$ appears in the $i$ th position. Therefore $\left(B(z, z)^{-1} v, v\right)=\frac{1}{(m-1) !(m+n-q)}\left|z_{n}^{m-1}\right|^{2}(\mathrm{I}+\mathrm{II})$, with

$$
\begin{aligned}
& \mathrm{I}=\left(1-|z|^{2}\right)^{-q} \sum_{j=q}^{n}\left|z_{j}\right|^{2} \\
& \mathrm{II}=q !\left(1-|z|^{2}\right)^{-q-1} \sum_{j=q}^{n}\left(z_{j} \sum_{i=1}^{q-1} e_{1} \wedge \cdots \wedge \overline{z_{i}} z \wedge \cdots \wedge e_{j}\right. \\
& \left.\quad+\left|z_{j}\right|^{2} e_{1} \wedge \cdots \wedge e_{q-1} \wedge z, w\right)
\end{aligned}
$$


moreover,

$$
\begin{aligned}
& \sum_{j=q}^{n}\left(\sum_{i=1}^{q-1} z_{j} e_{1} \wedge \cdots \wedge \overline{z_{i}} z \wedge \cdots \wedge e_{j}, w\right) \\
& =\frac{1}{q !} \sum_{j=q}^{n} \sum_{i=1}^{q-1}\left|z_{j}\right|^{2}\left|z_{i}\right|^{2} \\
& =\frac{1}{q !}\left(\sum_{j=q}^{n}\left|z_{j}\right|^{2}\right)\left(\sum_{i=1}^{q-1}\left|z_{i}\right|^{2}\right) \\
& \sum_{j=q}^{n}\left|z_{j}\right|^{2}\left(e_{1} \wedge \cdots \wedge e_{q-1} \wedge z, w\right) \\
& =\sum_{j=q}^{n} \sum_{k=q}^{n}\left|z_{j}\right|^{2}\left(e_{1} \wedge \cdots \wedge e_{q-1} \wedge z, e_{1} \wedge \cdots \wedge e_{q-1} \wedge e_{k}\right) \\
& =\frac{1}{q !} \sum_{j=q}^{n} \sum_{k=q}^{n}\left|z_{j}\right|^{2}\left|z_{k}\right|^{2}=\frac{1}{q !}\left(\sum_{j=q}^{n}\left|z_{j}\right|^{2}\right)^{2},
\end{aligned}
$$

and the summation of the inner products in II is

$$
\frac{1}{q !}\left(\sum_{j=q}^{n}\left|z_{j}\right|^{2}\right)^{2}+\frac{1}{q !}\left(\sum_{j=q}^{n}\left|z_{j}\right|^{2}\right)\left(\sum_{i=1}^{q-1}\left|z_{i}\right|^{2}\right)=|z|^{2} \sum_{j=q}^{n}\left|z_{j}\right|^{2}
$$

Eventually we find

$$
\begin{aligned}
\left(B(z, z)^{-1} v, v\right) & =\frac{1}{(m-1) !(m+n-q)}\left|z_{n}^{m-1}\right|^{2} \\
& \cdot\left(\sum_{j=q}^{n}\left|z_{j}\right|^{2}\right)\left(\left(1-|z|^{2}\right)^{-q}+\left(1-|z|^{2}\right)^{-q-1}|z|^{2}\right) \\
& =\frac{1}{(m-1) !(m+n-q)}\left|z_{n}^{m-1}\right|^{2}\left(\sum_{j=q}^{n}\left|z_{j}\right|^{2}\right)\left(1-|z|^{2}\right)^{-q-1}
\end{aligned}
$$


which has a simpler form. The norm $\|v\|_{\nu}^{2}$ is given by

$$
\begin{aligned}
& b_{\nu} \frac{\pi^{n} \Gamma(\nu-q-1-n)}{\Gamma(\nu-q-1)} \frac{1}{(m-1) !(m+n-q)} \frac{(n-q)(m-1) !+m !}{(\nu-q-1)_{m}} \\
& =b_{\nu} \frac{\pi^{n} \Gamma(\nu-q-1-n)}{\Gamma(\nu-q-1)} \frac{1}{(m-1) !(m+n-q)} \frac{(n-q+m)(m-1) !}{(\nu-q-1)_{m}} \\
& =\frac{1}{(\nu-1-n)(\nu-q)_{m-1}} .
\end{aligned}
$$

Thus we have:

Theorem 4.8. Suppose $1<q \leq n$. The following expansion holds:

$$
\begin{aligned}
& \otimes^{q} B(z, w)(1-(z, w))^{-\nu} \\
& =I+\sum_{m=1}^{\infty}(\nu-q)_{m} K_{-m \varepsilon_{n}+\varepsilon_{1}+\cdots+\varepsilon_{q}} \\
& \quad+\sum_{m=1}^{\infty}(\nu-1-n)(\nu-q)_{m-1} K_{-m \varepsilon_{n}+\varepsilon_{1}+\cdots+\varepsilon_{q-1}+\varepsilon_{n}}
\end{aligned}
$$

if $q<n$. For $q=n$ we have the known expansion for the weighted Bergman kernel

$$
\begin{aligned}
\otimes^{n} B(z, w)(1-(z, w))^{-\nu} & =(1-(z, w))^{-(\nu-n-1)} \\
& =\sum_{m=0}^{\infty}(\nu-n-1)_{m} \frac{1}{m !}(z, w)^{m} .
\end{aligned}
$$

Theorem 4.9. Let $1<q<n$. The representation $\mathcal{P} \otimes \wedge^{q} V$ is reducible if and only if $\nu=n+1$ or $\nu \leq q$ is an integer, in which case the composition factors $0=M_{0} \subset M_{1} \subset M_{2}=\mathcal{P} \otimes \wedge^{p} V^{\prime}$ are described as follows:

$$
M_{1}=\sum_{m=0}^{\infty} V^{-m \varepsilon_{n}+\varepsilon_{1}+\cdots+\varepsilon_{q}}
$$

if $\nu=n+1$;

$$
M_{1}=V^{\varepsilon_{1}+\cdots+\varepsilon_{q}}+V^{\varepsilon_{1}+\cdots+\varepsilon_{q-1}}
$$

if $\nu=q$; and

$$
M_{1}=\sum_{0 \leq m \leq q-\nu} V^{-m \varepsilon_{n}+\varepsilon_{1}+\cdots+\varepsilon_{q}}+\sum_{1 \leq m \leq q-\nu+1} V^{-m \varepsilon_{n}+\varepsilon_{1}+\cdots+\varepsilon_{q-1}+\varepsilon_{n}}
$$

if $\nu<q$. 


\section{References}

[1] W. Arveson, Subalgebras of $C^{*}$-algebras III: Multivariable operator theory, Acta Math., 181(2) (1998), 159-228, MR 1668582, Zbl 0952.46035.

[2] R.G. Douglas and V.I. Paulsen, Hilbert Modules over Function Algebras, Pitman Research Notes, 2, London, 1989, MR 1028546, Zbl 0686.46035.

[3] J. Faraut and A. Koranyi, Function spaces and reproducing kernels on bounded symmetric domains, J. Funct. Anal., 88 (1990), 64-89, MR 1033914, Zbl 0718.32026.

[4] R. Howe and E.-C. Tan, Homogeneous functions on light cones: The infinitesimal structure of some degenerate principal series representations, Bull. Amer. Math. Soc. (N.S.), 28(1) (1993), 1-74, MR 1172839, Zbl 0794.22012.

[5] L.K. Hua, Harmonic Analysis of Functions of Several Complex Variables in the Classical Domains, American Mathematical Society, Providence, Rhode Island, 1963, MR 0171936, Zbl 0112.07402.

[6] B. Ørsted and G. Zhang, Reproducing kernels and composition series for spaces of vector-valued holomorphic functions on tube domains, J. Funct. Anal., 124 (1994), 181-204, MR 1284609, Zbl 0819.46017.

[7] _ Reproducing kernels and composition series for spaces of vector-valued holomorphic functions, Pacific J. Math., 171(2) (1995), 493-510, MR 1372241, Zbl 0857.32011.

[8] J. Peetre and G. Zhang, Invariant Cauchy-Riemann operator and realization of relative discrete series of line bundle over the unit ball of $\mathbf{C}^{n}$, Michigan Math. J., 45 (1998), 387-397, MR 1637682, Zbl 0957.43004.

[9] W. Rudin, Function Theory in the Unit Ball of $\mathbb{C}^{n}$, Grundlehren Math. Wiss., 241, Springer-Verlag, 1980, MR 0601594, Zbl 0495.32001.

[10] W. Rühl, Field representations of the conformal group with continuous mass spectrum, Comm. Math. Phys., 30 (1973), 287-302, MR 0368634, Zbl 0257.22019.

[11] G. Shimura, Invariant differential operators on Hermitian symmetric spaces, Ann. Math., 132 (1990), 237-272, MR 1070598, Zbl 0718.11020.

[12] N. Wallach, Polynomial differential operators associated with Hermitian symmetric spaces, in 'Representation Theory of Lie Groups and Lie Algebras', Proceedings of Fuji-Kawaguchiko Conference 1990 (S. Sano., T. Kawazoe, T. Oshima, eds.), World Scientific Singapore, 1992, 76-95, MR 1190751.

[13] G. Zhang, Nearly holomorphic functions and relative discrete series of weighted $L^{2}$ spaces on bounded symmetric domains, J. Math. Kyoto Univ., 42(2) (2002), 207-221, MR 1966833.

[14] _ A weighted Plancherel formula II, the case of a ball, Studia Math., 102 (1992), 103-120, MR 1169281, Zbl 0811.43003.

Received April 15, 2003 and revised September 8, 2003.

Department of EngineERING

Physics AND Mathematics

UNIVERSITY OF KARLSTAD

S-651 88 KARLSTAD

SWEDEN 
E-mail address: stephen.hwang@kau.se

INSTITUTIONEN FÖR NATURVETENSKAP

ÖREBRo UNIVERSITET

ÖREBRo

SWEDEN

E-mail address: Yang.liu@nat.oru.se

Department of Mathematics

Chalmers University of Technology and Gothenburg University GothenBurG

SWEDEN

E-mail address: genkai@math.chalmers.se 